\title{
On the significance of borders: the emergence of endogenous dynamics
}

\author{
Ingrid Kubin ${ }^{1}$ D $\cdot$ Laura Gardini ${ }^{2}$
}

Received: 13 May 2020 / Accepted: 25 September 2020 / Published online: 5 November 2020

(C) The Author(s) 2020

\begin{abstract}
We propose a prototype model of market dynamics in which all functional relationships are linear. We take into account three borders, defined by linear functions, that are intrinsic to the economic reasoning: non-negativity of prices; downward rigidity of capacity (depreciation); and a capacity constraint for the production decision. Given the linear specification, the borders are the only source for the emerging of cyclical and more complex dynamics. In particular, we discuss centre bifurcations, border collision bifurcations and degenerate flip bifurcations-dynamic phenomena the occurrence of which are intimately related to the existence of borders.
\end{abstract}

Keywords Capacity constraint - Depreciation constraint - Nonnegativity constraint · Piecewise smooth system · Border collision bifurcation · Centre bifurcation

\section{Introduction}

In the aftermath of the recent financial crises, economic models that consider constraints and boundaries have become increasingly popular, in particular constraints in the financial markets: liquidity constraints, borrowing constraints, credit constraints, collateral constraints, etc. These constraints primarily reflect imperfections in the financial markets. However, there are other constraints with an even longer, albeit less noted, history that do not involve any market imperfections: e.g. capacity constraints, which bind production decisions, feature prominently in industrial organization models, but also in business cycle models in the tradition of Samuelson and Hicks; or incentive and participation constraints that play a central role in contract theory. And

\footnotetext{
$\bowtie \quad$ Ingrid Kubin

ingrid.kubin@wu.ac.at

Laura Gardini

laura.gardini@uniurb.it

1 Department of Economics, WU Vienna University of Economics and Business, Vienna, Austria

2 Department of Economics, Society, Politics, University of Urbino, Urbino, Italy
} 
there are even more fundamental constraints, such as budget constraints and nonnegativity constraints for quantities and prices.

Recently, considering inequality constraints in dynamic models, Rendahl (2017) pointed out that this asks for specific analytic methods. ${ }^{1}$ Even less known is another analytic implication: the mere existence of boundaries creates the potential for cyclical and even chaotic dynamic time paths. This neglect is astonishing since the financial crises has powerfully shown that the stability of equilibria and fixed points cannot be taken as granted. Instead, the analysis of out of equilibrium dynamics should be of prime importance.

In the following, we show dynamic phenomena that directly derive from the consideration of boundaries. For that purpose, we propose a very simple model that involves boundaries, but is linear otherwise and continuous. Thus, any complex dynamics cannot be attributed to nonlinear functional specifications that might be considered as arbitrary; it directly derives from the boundaries, crossing which the linear system changes definition. In particular, we present a very simple model of market dynamics in the Marshallian tradition. Quantity decisions - on current output and on capacity adjustments - depend on a comparison of the relevant demand and supply price. Output is sold against the market demand function at a market clearing price. On purpose, we assume all functional relationships to be linear. In addition, we explicitly take into account three borders that are intrinsic to the economic reasoning: a non-negativity constraint for prices; a downward rigidity of capacity (depreciation) and a capacity constraint for the production decision.

The study of the dynamics which are involved in piecewise linear two-dimensional maps is not a new research subject. It started several years ago, and important early contributions include Gumowski and Mira (1980), Nusse and Yorke (1992), Mira et al. (1996), and Gardini (1992). However, this area is not so well known and developed. Also two influential surveys on analytic tools for economic dynamics, Barnett et al. (2015) and Grandmont (2008), present only tools for smooth functions. Many properties are still to be investigated and in the present paper we apply most recent analytic tools to a prototype economics model.

There are also some contributions in economics that do already involve borders and not only with piecewise smooth functions, occasionally also with discontinuous specification of the central dynamic process. In business cycle theory, models in the tradition of Hicks and Kaldor with a "floor" and a "ceiling" (Hommes 1995; Sushko et al. 2003, 2010; Gardini et al. 2006) as well as regime switching models (Tramontana et al. 2010); in growth theory (Matsuyama 1999, 2007; Boehm and Kaas 2000; Kaas and Zink 2007; Tramontana et al. 2011a), in industrial organization theory, managerial economics and competition models (Kopel 1996; Laugesen and Mosekilde 2006; Bischi and Lamantia 2012; Bischi et al. 2012; Schmitt et al. 2017), models of the so-called New Economic Geography and industry location (Currie and Kubin 2006; Commendatore et al. 2008a, b, 2020; Agliari et al. 2011; Schmitt et al. 2018), and also in macroeconomic models with financial markets (Caballé et al. 2006; Tramontana et al. 2011b; Matsuyama 2013; Sushko et al. 2014, 2016; Kubin and Zörner 2019)

\footnotetext{
1 In his work, the author aims to bridge the gap between dynamic programming techniques (which are reliable but often slow) and Euler equation-based methods (which are known to be faster but have difficult convergence properties).
} 
borders play a crucial role. In fact, in his survey on macro-dynamics, Gomes (2006) identifies the piecewise smooth specification as one of five influential model types.

Moreover, improvements in the dynamic tools which can be used to investigate one-dimensional piecewise smooth systems recently appeared (Sushko et al. 2015, 2016), and also two-dimensional piecewise smooth models in economic applications have been investigated (Sushko et al. 2017; Gardini et al. 2018).

In our contribution, the focus relies on the connection that exists between the borders, which have a genuine economic rational, and the dynamic specification. As already remarked, we present a simple prototype model of the market dynamics in the Marshallian tradition which involves three borders and in which all functional relations are linear. ${ }^{2}$ Time is discrete and structured by the production period. Decisions on output and capacity depend (linearily) on the difference between the corresponding supply and demand prices. At the end of the production period, output is sold at against a (linear) market demand, and the resulting market prices feed back upon quantity decisions. Output, capacity and market prices are subject to a non-negativity constraint, output is bounded by a capacity constraint, and capacity reductions cannot exceed depreciation. The resulting model is piecewise linear in two dimensions (output and capacity) and involves three borders. In particular we discuss three dynamic phenomena, the occurrence of which are intimately related to the existence of borders: centre bifurcations, border collision bifurcations and degenerate flip bifurcations. The emerging dynamics may be complex and in our analysis we pay special attention to the role of the borders in shaping the resulting dynamics.

The plan of the paper is as follows. In Sect. 2, we present the model and define the different regions in the phase space in which the continuous map changes its definition. In Sect. 3 we describe the dynamic behaviour generated by this map. In particular, Sect. 3.1 completely characterises the unique fixed point of the map, showing that it loses stability via a centre bifurcation (see Sushko and Gardini 2008) that always (i.e. independently on the parameters of the map) occurs through a rotation of period six and leads to a stable period-6 cycle (belonging to an attracting closed invariant curve, saddle-node connection of a pair of 6-cycles). In addition, we show that this period-6 cycle loses stability through a so-called secondary centre bifurcation leading to annular chaotic areas (see Gardini 1994; Gardini et al. 2011) as well as to chaotic areas in one piece (see Mira et al. 1996; Fournier-Prunaret et al. 1997; Maistrenko et al. 1998; Sushko et al. 1999). In Sect. 3.2, we shall describe two additional dynamic phenomena, which are caused by the existence of the borders. In particular, we describe the so-called border collision bifurcations [following the terminology introduced by Nusse and Yorke (1992)], which occur if some periodic point of a cycle merges with a border line; and we describe the peculiar character of a flip bifurcation which, due to the linearity of the functions, is degenerate (Sushko and Gardini 2010). Section 4 concludes.

\footnotetext{
2 The model is inspired by Currie and Kubin (1997), a nonlinear version is studied in Kubin and Gardini (2013).
} 


\section{The basic framework}

In the following, we develop a very simple prototype model of the dynamic interaction between the decisions on output and capacity adjustment and the price determination in a competitive market for a single homogenous commodity. We choose an unusual approach. After introducing the assumption on production technology, we discuss the borders that play an important role in our model and we trace explicitly the elements of the model that are already implied by the specification of the borders. Only later whenever necessary, we introduce the behavioural framework that rests upon ideas taken from evolutionary economics.

Production technology is assumed to be as simple as possible. Production takes time (one period); and requires as input one unit of fixed capital (one machine) and a constant quantity of labour and raw material per unit of the final output. While the required quantity of labour and raw material can be acquired instantaneously, the stock of machines can only be increased with a gestation lag of one period. It depreciates irrespective of use at a constant rate $\delta$ per period. Once installed, machines are specific to the particular industry and thus their use involves no opportunity costs. Entrepreneurs decide upon capacity adjustments and production output that is sold in a competitive market. Given that we are focussing on the (partial) analysis of a single market, we take the prices for the variable inputs and for new machines, denoted by $v$ and $m$ resp., as fixed. In addition, we assume that entrepreneurs can finance any cost at a-given-interest rate, denoted by $i$.

The first border that we observe is the non-negativity constraint for prices. We assume that there exists a maximum quantity that can be sold at a non-negative price (denoted by $a$ ) and this assumption splits the phase space into two halves. For $q_{t} \geq a$ the constraint implies that in this region $p_{t}=0$ holds (where $q_{t}$ denotes the quantity sold at time $t$ and $p_{t}$ denotes the price); for $0 \leq q_{t}<a$ we have to specify a demand function, i.e. $p_{t}=P\left(q_{t}\right)$ with $\frac{\mathrm{d} P}{\mathrm{~d} q_{t}}<0$. Note that the demand function allows reducing the phase space to a two dimensional one in output and capacity.

The second border that we take into consideration is the capacity constraint, which follows from the following reasoning: production requires fixed capital (machines) as input and the stock of fixed capital (machines) cannot be adjusted instantaneously. Therefore, the current production decision is restricted by the currently existing capacity. We allow for a production lag of one period and denote with $\widetilde{q}_{t+1}$ the intended output for period $t+1$. The capacity constraint is binding, whenever the intended output is higher than the existing capacity, denoted by $k_{t}$, i.e. whenever $\widetilde{q}_{t+1} \geq k_{t}$. For that case, the quantity dynamics is already implied by the constraint itself and is given by $q_{t+1}=k_{t}$.

The third border in our model concerns the downward rigidity of capacity adjustment. Assuming that machines once installed are specific to the industry, a capacity reduction cannot exceed the depreciation. We allow for a one period lag in capacity adjustment (i.e. a gestation lag for new machines) and denote the intended capacity for period $t+1$ by $\widetilde{k}_{t+1}$. The depreciation constraint is binding, whenever the intended capacity reduction is higher than the depreciation, i.e. whenever $\widetilde{k}_{t+1} \leq k_{t}(1-\delta)$. Again, the capacity dynamics for that case is already implied by the constraint and given by $k_{t+1}=k_{t}(1-\delta)$. 
Therefore, if a border is binding, it is the border that implies the dynamic (or static) outcome for that case-roughly spoken, half of the dynamics is already given by the borders.

In order to proceed, we have to specify behavioural rules for the intended quantity and the intended capacity. In doing so, we succeed in relating the borders to the phase space as well as in specifying the dynamics for the cases in which no border is binding.

For modelling entrepreneurial behaviour we follow the line suggested in Kubin and Gardini (2013) and start from the following observations that are well documented in managerial, evolutionary, behavioural economics as well as in systems dynamics (for overviews, see e.g. Dosi et al. 2008; Aramburo et al. 2012). In an environment characterised by nonlinearities, feedback mechanisms and delays — and our model will definitely fall in this class - the scope for learning the underlying model is limited [see Dosi et al. (2008), who extensively document various impediments to learn] and economic agents tend to base their decisions on incomplete and inaccurate mental models or to recur to simple heuristics. In their famous survey on heuristics and biases, Tversky and Kahneman (1974, p. 185) note that "in many situations, people make estimates by starting from an initial value that is adjusted to yield the final answer" and that "adjustments are typically insufficient". Sterman (1989), in his seminal paper, identifies this anchoring and (insufficient) adjustment behaviour as typical heuristic used in capacity adjustment decisions (with the status quo being quite naturally used as anchor) and provides evidence for its intended rationality (similar also in his more recent study, Sterman et al. 2007). Aramburo et al. (2012) review results from a vast number of laboratory experiments on decision making in complex and dynamic environments. They conclude that economic agents tend to ignore delays and that in complex environments "experimental data fit a decision rule based on the anchoring and adjustment heuristic" (Aramburo et al. 2012, p. 98).

For our model, we follow this line of reasoning and assume that entrepreneurs base their decisions on an anchoring and (insufficient) adjustment heuristic, where the status quo is used as anchor and where the (insufficient) adjustment follows from market observations. In order to be more precise, note that in a competitive market with many (homogeneous) competitors a single entrepreneur would have difficulties to observe total market capacity, total market supply and total market demand, but the own capacity, the own quantity produced and the market price for the output can be easily observed. This suggests a Marshallian perspective on managerial decision heuristics. Observable individual quantities serve as anchor and they are (insufficiently) adjusted-following a Marshallian tradition-whenever the current market price is not equal to the relevant Marshallian supply price.

First, we model the output decision. Given that machines, once installed, are specific to the particular industry, the costs of the inherited machines are sunk, and a Marshallian short-run supply price is equal to the effective variable cost $v(1+i)$. As noted above, we assume $v(1+i)$ to be constant. The intended quantity is thus higher the higher the current production (anchoring behaviour) and the higher the current price (adjustment behaviour); therefore, $\widetilde{q}_{t+1}=\widetilde{Q}\left(q_{t}, p_{t}\right)$ with $\frac{\partial \widetilde{Q}}{\partial q_{t}}>0$ and $\frac{\partial \widetilde{Q}}{\partial p_{t}}>0$. Using the demand function, results in $\widetilde{q}_{t+1}=\widetilde{Q}\left(q_{t}, P\left(q_{t}\right)\right)$. This specification allows us to relate the second border, the capacity constraint, to the phase 
space. On the border $\widetilde{q}_{t+1}=k_{t}$ holds or $k_{t}=\widetilde{Q}\left(q_{t}, P\left(q_{t}\right)\right)$ with $\frac{\mathrm{d} k_{t}}{\mathrm{~d} q_{t}}=\frac{\partial \widetilde{Q}}{\partial q_{t}}+\frac{\partial \widetilde{Q}}{\partial P} \frac{\mathrm{d} P}{\mathrm{~d} q_{t}}$. Since $\frac{\mathrm{d} P}{\mathrm{~d} q_{t}}<0$ for $0 \leq q_{t}<a$, the second boundary, the capacity constraint, can have a positive or negative slope in that region, depending on the relative strength of the anchoring and adjustment behaviour. In contrast, $\frac{\mathrm{d} P}{\mathrm{~d} q_{t}}=0$ holds for $q_{t} \geq a$; in that region, the capacity constraint has a positive slope. In addition, the behavioural rule implies the dynamic outcome for the case in which the capacity constraint is not binding: $q_{t+1}=\widetilde{Q}\left(q_{t}, P\left(q_{t}\right)\right)$ if $k_{t}>\widetilde{Q}\left(q_{t}, P\left(q_{t}\right)\right)$.

Second, we model the capacity adjustment decision. The corresponding long-run supply price, denoted by $c$, is given as follows:

$$
c=v(1+i)+m(\delta+i)
$$

where $m(\delta+i)$ represents the ex ante per period costs of buying and installing a machine instead of investing in the financial market. We assume that the long-run supply price is constant as well and that $c<a$ holds.

Entrepreneurs intend to increase capacity whenever the current market price is higher than the long-run supply price. The higher the current capacity (anchoring behaviour) and the higher the current price (adjustment behaviour), the higher the intended capacity; therefore, $\widetilde{k}_{t+1}=\widetilde{K}\left(k_{t}, p_{t}\right)$ with $\frac{\partial \widetilde{K}}{\partial k_{t}}>0$ and $\frac{\partial \widetilde{k}}{\partial p_{t}}>0$. Using the demand function, results in $\widetilde{k}_{t+1}=\widetilde{K}\left(k_{t}, P\left(q_{t}\right)\right)$. This specification allows us to relate the third border, the depreciation constraint, to the phase space. On the border $\widetilde{k}_{t+1}=k_{t}(1-\delta)$ holds or $k_{t}(1-\delta)=\widetilde{K}\left(k_{t}, P\left(q_{t}\right)\right)$. For determining the slope observe $(1-\delta) \mathrm{d} k_{t}=\frac{\partial \widetilde{K}}{\partial k_{t}} \mathrm{~d} k_{t}+\frac{\partial \widetilde{K}}{\partial P} \frac{\mathrm{d} P}{\mathrm{~d} q_{t}} \mathrm{~d} q_{t}$; thus $\frac{\mathrm{d} k_{t}}{\mathrm{~d} q_{t}}=\frac{\frac{\partial \widetilde{K}}{\partial P} \frac{\mathrm{d} P}{\mathrm{~d} q_{t}}}{1-\delta-\frac{\partial \widetilde{K}}{\partial k_{t}}}$. Similar to the capacity constraint also the third boundary, the depreciation constraint, may have a positive or negative slope in the area $0 \leq q_{t}<a$. Instead, for $q_{t} \geq a$ the depreciation constraint has a zero slope. Again, the behavioural rule implies the dynamic outcome for the case in which the depreciation constraint is not binding: $k_{t+1}=\widetilde{K}\left(k_{t}, P\left(q_{t}\right)\right)$ if $\widetilde{K}\left(k_{t}, P\left(q_{t}\right)\right)>k_{t}(1-\delta)$.

We thus have illustrated how the definition of the borders and the specification of the behavioural rules interact in specifying the full dynamic model. Both are necessary and the borders - that are intrinsic to the model and generally much less disputed than the behavioural rules-contribute much to this specification.

The analysis of the dynamics generated by this model requires a more explicit specification of the behavioural rules. On purpose-and in contrast to Kubin and Gardini (2013) — we shall use linear specifications; therefore, any nonlinear dynamics comes from the - much less disputed - borders, and not from a nonlinear specification of the behavioural rules that might be considered arbitrary.

With a linear demand function and observing the non-negativity constraint the market clearing price, $p_{t}$, is given as:

$$
p_{t}= \begin{cases}0 & \text { for } q_{t}=0 \\ a-q_{t} & \text { for } 0<q_{t} \leq a \\ 0 & \text { for } q_{t}>a\end{cases}
$$


With a linear specification the intended quantity is given as:

$$
\tilde{q}_{t+1}=q_{t}+\mu\left(p_{t}-v(1+i)\right)
$$

For $0<q_{t} \leq a$ the second boundary, the capacity constraint (shown in magenta in Fig. 1), can be written as $k_{t}=q_{t}+\mu\left(a-q_{t}-v(1+i)\right)$, i.e. $k_{t}=q_{t}(1-\mu)+\mu(a-$ $v(1+i))=: f_{1}\left(q_{t}\right)$, while for $q_{t}>a$ the second boundary (also shown in magenta in Fig. 1) is $k_{t}=q_{t}-\mu \nu(1+i)=: f_{2}\left(q_{t}\right)$.

Thus, we have the following output dynamics:

$$
q_{t+1}= \begin{cases}f_{1}\left(q_{t}\right)=q_{t}(1-\mu)+\mu(a-v(1+i)) & \text { if } 0<q_{t} \leq a \text { and } k_{t} \geq f_{1}\left(q_{t}\right) \\ f_{3}\left(k_{t}\right)=k_{t} & \text { if } 0<q_{t} \leq a \text { and } k_{t}<f_{1}\left(q_{t}\right) \\ f_{2}\left(q_{t}\right)=q_{t}-\mu \nu(1+i) & \text { if } q_{t}>a \quad \text { and } k_{t} \geq f_{2}\left(q_{t}\right) \\ f_{3}\left(k_{t}\right)=k_{t} & \text { if } q_{t}>a \quad \text { and } k_{t}<f_{2}\left(q_{t}\right)\end{cases}
$$

With a linear specification, the intended capacity is given as:

$$
\widetilde{k}_{t+1}=k_{t}+\sigma\left(p_{t}-c\right)
$$

For $0<q_{t} \leq a$ the third border, the depreciation constraint (shown in green in Fig. 1), can be written as $k_{t}(1-\delta)=k_{t}+\sigma\left(a-q_{t}-c\right)=: g_{1}\left(q_{t}, k_{t}\right)$ or $(1-\delta) k_{t}=g_{1}\left(q_{t}, k_{t}\right)$ or $k_{t}=\frac{\sigma}{\delta} q_{t}+\frac{\sigma}{\delta}(c-a)$. For $q_{t}>a$ the third border (also shown in green in Fig. 1) is $k_{t}(1-\delta)=k_{t}-\sigma c:=g_{2}\left(k_{t}\right)$ or $k_{t}=\frac{\sigma}{\delta} c$.

Thus we have the following capacity dynamics:

$$
k_{t+1}= \begin{cases}g_{1}\left(q_{t}, k_{t}\right)=k_{t}-\sigma q_{t}+\sigma(a-c) & \text { if } 0<q_{t} \leq a \text { and } g_{1}\left(q_{t}, k_{t}\right) \geq(1-\delta) k_{t} \\ g_{3}\left(k_{t}\right)=(1-\delta) k_{t} & \text { if } 0<q_{t} \leq a \text { and } g_{1}\left(q_{t}, k_{t}\right)<(1-\delta) k_{t} \\ g_{2}\left(k_{t}\right)=k_{t}-\sigma c & \text { if } q_{t}>a \text { and } g_{2}\left(q_{t}, k_{t}\right) \geq(1-\delta) k_{t} \\ g_{3}\left(k_{t}\right)=(1-\delta) k_{t} & \text { if } q_{t}>a \quad \text { and } g_{2}\left(q_{t}, k_{t}\right)<(1-\delta) k_{t}\end{cases}
$$

So the economic model is ultimately described by (4) and (6), i.e. by a two-dimensional continuous piecewise linear map in the plane, say $\left(q_{t+1}, k_{t+1}\right)=T\left(q_{t}, k_{t}\right)$, where the definition of $T$ changes (although continuously) at borders (in the phase plane $(q, k)$ ) that are due to the economically motivated constraints.

As long as the parameters satisfy the inequality $\frac{\sigma c}{\delta}<a-\mu \nu(1+i)$, the positive area of the phase plane $(q, k)$ is represented by 5 different regions, where the map takes different definitions, as shown in Fig. 1a, while for $\frac{\sigma c}{\delta}>a-\mu \nu(1+i)$ we have 7 regions, as shown in Fig. 1 b.

The definition of the map is given, in explicit form in each region, by

$$
\begin{aligned}
& R_{1}:\left[\begin{array}{l}
q_{t+1} \\
k_{t+1}
\end{array}\right]=\left[\begin{array}{c}
f_{1}\left(q_{t}\right) \\
g_{1}\left(q_{t}, k_{t}\right)
\end{array}\right], \quad R_{2}:\left[\begin{array}{l}
q_{t+1} \\
k_{t+1}
\end{array}\right]=\left[\begin{array}{l}
f_{2}\left(q_{t}\right) \\
g_{2}\left(k_{t}\right)
\end{array}\right] \\
& R_{3}:\left[\begin{array}{l}
q_{t+1} \\
k_{t+1}
\end{array}\right]=\left[\begin{array}{c}
f_{3}\left(k_{t}\right) \\
g_{1}\left(q_{t}, k_{t}\right)
\end{array}\right], \quad R_{4}:\left[\begin{array}{l}
q_{t+1} \\
k_{t+1}
\end{array}\right]=\left[\begin{array}{l}
f_{3}\left(k_{t}\right) \\
g_{2}\left(k_{t}\right)
\end{array}\right], \quad R_{5}:\left[\begin{array}{l}
q_{t+1} \\
k_{t+1}
\end{array}\right]=\left[\begin{array}{l}
f_{3}\left(k_{t}\right) \\
g_{3}\left(k_{t}\right)
\end{array}\right] \\
& R_{6}:\left[\begin{array}{l}
q_{t+1} \\
k_{t+1}
\end{array}\right]=\left[\begin{array}{l}
f_{1}\left(q_{t}\right) \\
g_{3}\left(k_{t}\right)
\end{array}\right], \quad R_{7}:\left[\begin{array}{l}
q_{t+1} \\
k_{t+1}
\end{array}\right]=\left[\begin{array}{l}
f_{2}\left(q_{t}\right) \\
g_{3}\left(k_{t}\right)
\end{array}\right]
\end{aligned}
$$


(a)

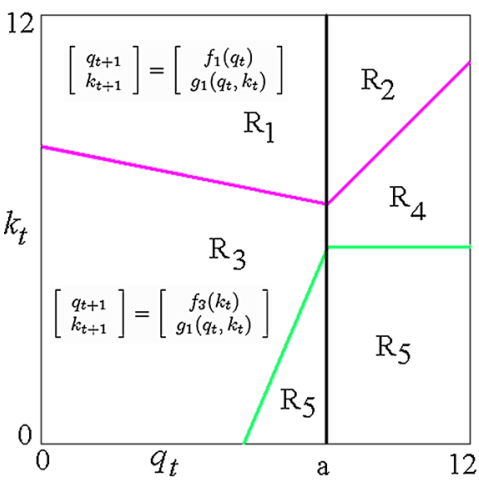

(b)

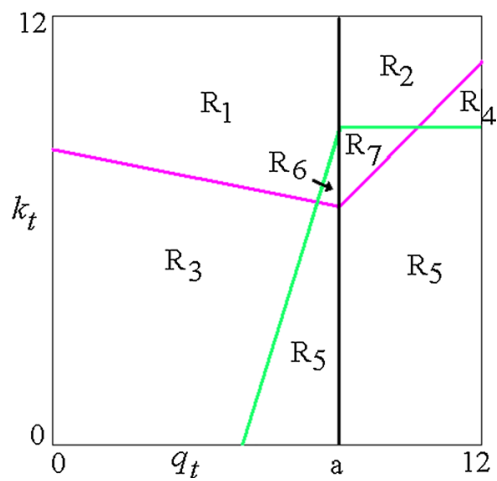

Fig. 1 Qualitative description of the different regions in the phase space. In a for $\frac{\sigma c}{\delta}<a-\mu \nu(1+i), 5$ regions. In $\mathbf{b}$ for $\frac{\sigma c}{\delta}>a-\mu \nu(1+i), 7$ regions (color figure online)

We notice that in the rightmost regions where $q>a$ the variable $k_{t}$ necessarily decreases; therefore, an iterated point $\left(q_{t}, k_{t}\right)$ is forced to enter some region where $q<a$. In the region $R_{5}$ the map is a contraction, and such that a point $\left(q_{t}, k_{t}\right)$ is forced to enter the region $R_{3}$.

It is also easy to see that a fixed point cannot exist in the regions $R_{1}$ and $R_{6}$ so that let us look at the definition of the map in region $R_{3}$, given by $\left(q_{t+1}, k_{t+1}\right)=$ $\left(f_{3}\left(k_{t}\right), g_{1}\left(q_{t}, k_{t}\right)\right)=\left(k_{t}, k_{t}-\sigma q_{t}+\sigma(a-c)\right)$. This map has a unique fixed point given by $(q, k)=\left(k^{*}, k^{*}\right)$ where

$$
k^{*}=(a-c)=(a-v(1+i)-m(\delta+i))>0
$$

and thus the two-dimensional map $T$ has also a positive fixed point $(q, k)=$ $\left(k^{*}, k^{*}\right)=: K^{*}$ iff this point belongs to the region $R_{3}$.

Proposition 1 The system defined in (4) and (6) has a unique positive fixed point given by $K^{*}=\left(k^{*}, k^{*}\right)$, belonging to Region $R_{3}$.

To prove the proposition we have to show that the fixed point belongs to that region as we have already shown that no other fixed point exists in the other regions. First we notice that $k^{*}=(a-c)<a$ so that the fixed point always belong to the left side of the straight line determined by the constraint $a$. To show that the fixed point is always above the constraint separating the regions $R_{3}$ and $R_{5}$ we have to prove that the fixed point always satisfies $g_{1}\left(q_{t}, k_{t}\right) \geq(1-\delta) k_{t}$, and in fact it is $g_{1}\left(k^{*}, k^{*}\right)-(1-\delta) k^{*}=\delta(a-c)>0$. Then we have to prove that the fixed point is always below the constraint separating the regions $R_{1}$ and $R_{3}$, that means $f_{1}\left(k^{*}\right)>k^{*}$. And in fact we have $f_{1}\left(k^{*}\right)=k^{*}(1-\mu)+\mu(a-v(1+i))>k^{*}$ iff $-(a-c) \mu+\mu(a-v(1+i))>0$ iff $m(\delta+i)>0$ which is always satisfied. 


\section{Dynamic properties}

In a linear model cyclical or complex dynamics is only possible because of the existence of borders, at which the definition of the (linear) dynamic specification changes. In the following, we show how the borders shape the dynamic properties of our model. In particular, we discuss the dynamics implied by centre bifurcations, by border collision bifurcations and by degenerate flip bifurcations-i.e. dynamic phenomena in which borders play a crucial role.

\subsection{Centre bifurcation}

We start investigating the stability properties of the fixed point $K^{*}=\left(k^{*}, k^{*}\right)$. The Jacobian of the system defined in region $R_{3}$ is a constant matrix given by

$$
J_{3}=\left[\begin{array}{cc}
0 & 1 \\
-\sigma & 1
\end{array}\right]
$$

whose characteristic polynomial is given by $\mathcal{P}(\lambda)=\lambda^{2}-\lambda+\sigma$. The related eigenvalues $\lambda_{1,2}=\frac{1 \pm \sqrt{1-4 \sigma}}{2}$ are complex conjugated for $\sigma>1 / 4$. The fixed point can loose stability via a centre bifurcation, occurring when the complex eigenvalues become equal to +1 in modulus, which occurs for $\sigma=1$, that is when the complex conjugate eigenvalues are $\lambda_{1,2}=\frac{1}{2} \pm j \frac{\sqrt{3}}{2}$ which corresponds to a rational rotation with rotation number $\frac{1}{6}$ (of angle $\frac{2 \pi}{6}$ ). As shown in Sushko and Gardini (2008), a piecewise linear map undergoing a centre bifurcation leads to an invariant polygon, filled with invariant curves on which the trajectories are either all periodic or quasiperiodic, depending on the rotation number (rational or irrational, respectively), and all the trajectories in the polygon are stable but not attracting, i.e. not asymptotically stable. In our case for $\sigma=1$, an invariant polygon $P$ exists, bounded by six segments of straight lines, one segment must belong to the border of definition of the region $R_{3}$ and the other segments are given by its images under $T$. We can now prove the following

Proposition 2 Consider the system defined in (4) and (6).

(i) At $\sigma=1$ an attracting Polygon $P$ exists in Region $R_{3}$ filled with cycles of period 6, each single cycle is stable but not attracting.

(ii) Let $\sigma<1$ and $\mu<1$ then the unique fixed point $K^{*}$ is globally stable.

Having shown that for $\sigma=1$ the Jacobian matrix in region $R_{3}$ (to which the fixed point $K^{*}$ belongs) has complex eigenvalues equal to 1 in modulus with a rational rotation number $\frac{1}{6}$, to end the proof we have only to show that for $\sigma<1$ and $\mu<1$, when the fixed point is locally stable, it is globally attracting. To see this, we notice that when the fixed point is locally stable, we have an immediate basin of attraction $B_{i m}$ which is the widest region belonging to $R_{3}$ made up of points which are converging to the fixed point with a trajectory which is completely included in $R_{3}$, and we show that starting from any other point outside $B_{i m}$ we must necessarily enter $B_{i m}$ in a finite number of steps. In fact, the map defined in region $R_{1}$ is in triangular form, where the 
(a)

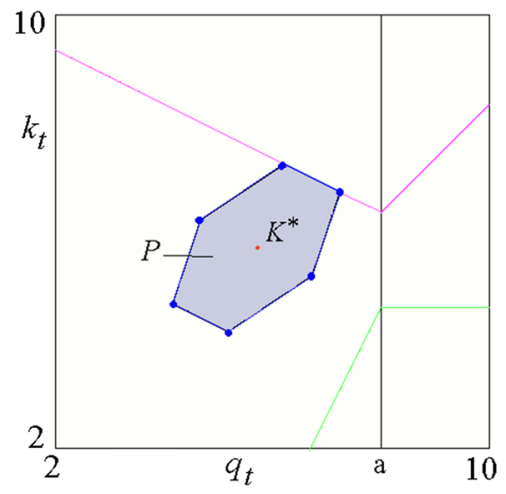

(b)

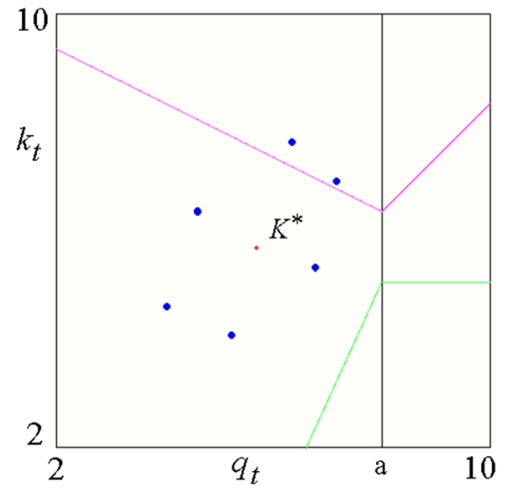

Fig. 2 In a centre bifurcation at $\mu=1.5, \delta=0.5$ and $\sigma=1$. In $\mathbf{b}$ a unique attracting six cycles exists, at $\sigma=1.1$

linear function $f_{1}\left(q_{t}\right)$ is a contraction for $\mu<1$ and tends to $q^{*}=a-v(1+i)<a$ while the variable $k_{t}$ is governed by $g_{1}\left(q_{t}, k_{t}\right)$ which leads to $g_{1}\left(q^{*}, k_{t}\right)=k_{t}-\sigma q^{*}+$ $\sigma(a-c)=k_{t}-\sigma(a-v(1+i))+\sigma(a-v(1+i)-m(\delta+i))=k_{t}-\sigma m(\delta+i)$ that is $k_{t+1}=k_{t}-\sigma m(\delta+i)$ which is decreasing. Thus any point $\left(q_{t}, k_{t}\right) \in R_{1}$ is necessarily mapped in region $R_{3}$ in a finite number of steps. We have already noticed above that any point belonging to the region $q>a$ is necessarily mapped to the region $q<a$, so that, from the properties of the maps defined in the other regions, in a finite number of steps the immediate basin $B_{i m}$ must be reached.

We remark that part (ii) of Proposition 2 is only a sufficient condition. That is, for $\sigma<1$, the stable fixed point may be globally stable also when $\mu>1$, but the proof cannot follow the steps used above, as the map in triangular form defined in region $R_{1}$ has the linear function $f_{1}\left(q_{t}\right)$ which is an expansion, and the points are pushed away from $q^{*}$.

What occurs after the centre bifurcation of the fixed point clearly depends on the combined effect of the functions defined mainly in the two regions $R_{3}$ and $R_{1}$. However, the rational rotation with rotation number $\frac{1}{6}$ suggests that also for $\sigma>1$, close to the bifurcation value, a unique attracting cycle of period 6 exists, that is, the infinitely many 6-cycles, stable but not attracting, leads to a unique 6-cycle attracting node and, as we shall see, after the centre bifurcation of the fixed point the dynamics may become quite soon complicated. An example of the centre bifurcation is shown in Fig. 2 at the following parameter values:

$$
a=8, \quad v=1, \quad m=2, \quad i=0.1
$$

which will be kept fixed in all our simulations in this work, and the parameter $c$ is computed as in (1).

Figure 2a shows the invariant polygon $P$ existing when the fixed point is a centre, at the bifurcation value (on the cuve $C_{1}$ in Fig. 3a). Before the bifurcation, being $\mu=0.95<1$, the fixed point is globally attracting. At the bifurcation value the 

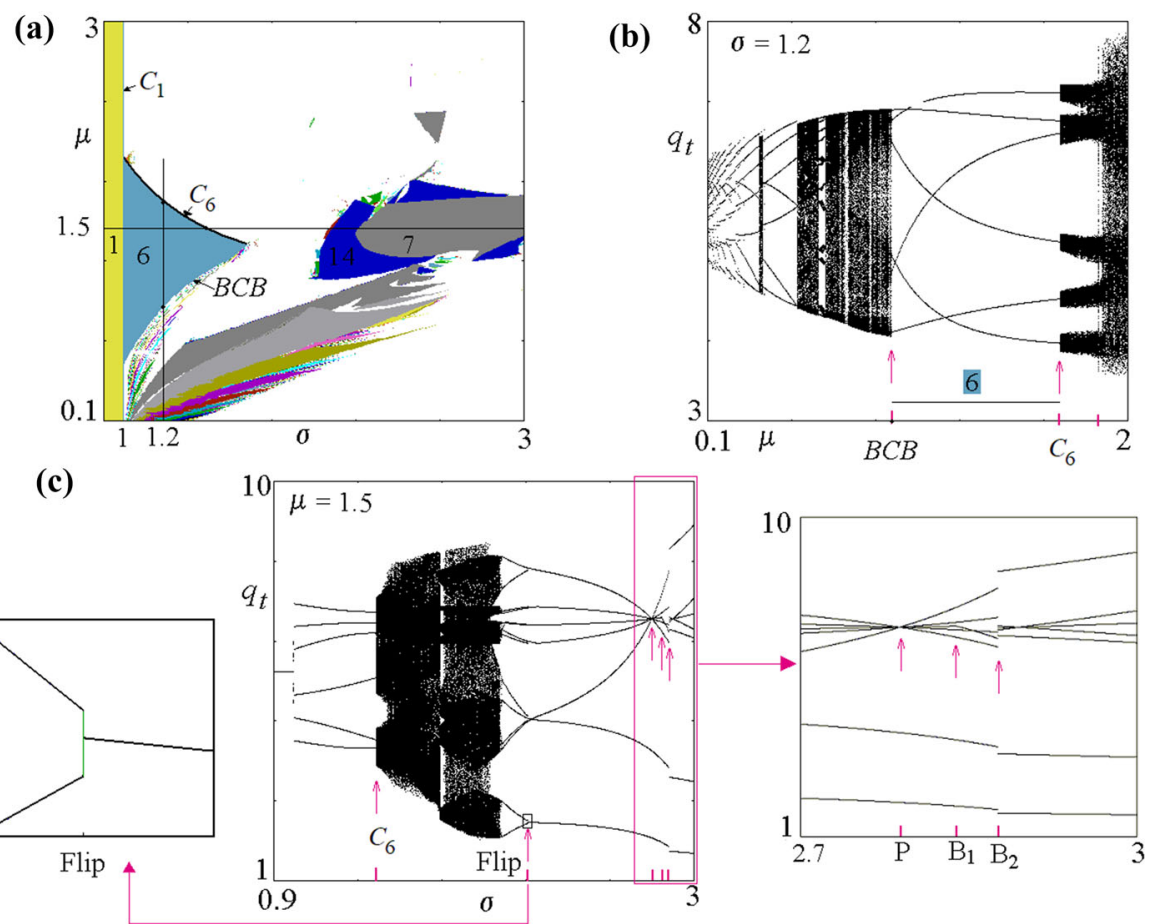

Fig. 3 In a two-dimensional bifurcation diagram in the parameter plane $(\sigma, \mu)$ at $\delta=0.5$ fixed. The centre bifurcation of the fixed point is labelled by $C_{1}$, while that of the 6-cycle by $C_{6}$. In $\mathbf{b}$ one-dimensional bifurcation diagram showing $q_{t}$ as a function of $\mu$ at $\sigma=1.2$ fixed, along the vertical path showin in (a). In c one-dimensional bifurcation diagram showing $q_{t}$ as a function of $\sigma$ at $\mu=1.5$ fixed, along the horizontal path shown in (a) (color figure online)

invariant polygon, included in region $R_{3}$, is bounded by six segments, one segment belongs to the curve defining a border of the region $R_{3}$, and the other segments are obtained by iteration of this segment by the map $T$ in this region. Any point external to the polygon is mapped into it in a finite number of iterations, and any point of the polygon (boundary included) belongs to six cycle (i.e. is periodic of period 6).

After the bifurcation, as we can see in Fig. 2b, only one attracting cycle is left, with two periodic points belonging to region $R_{1}$ and four periodic points belonging to region $R_{3}$. In Fig. 2 b only the attracting cycle is shown, however it is worth to recall that (due to the continuity of the map) the result of the centre bifurcation is a pair of 6-cycles, one of which is always a saddle while the other may be locally attracting (as in Fig. 2b) or a repelling node. When the 6-cycle is attracting we may have a closed curve connecting the saddle with the attracting cycle, made up of the unstable set of the 6-cycle saddle, while the stable set of the saddle is an invariant set which belongs to the frontier of the six disjoint fixed points for the sixth iterate of the map, i.e. for $T^{6}$. An example will be given below (in Fig. 4a).

In order to further investigate the dynamic properties, we will refer to the bifurcation diagrams in Fig. 3 (that are drawn for $\delta=0.5$ ). Figure 3a is a two-dimensional 
bifurcation diagram in the parameter plane $(\sigma, \mu)$. Different colors correspond to attracting cycles of different periods, up to period 45 . White points denote either a chaotic attractor or a different attracting set, not chaotic, as a cycle with periodicity higher than 45 or a closed invariant curve. We can see that at $\sigma=1$ (vertical line in Fig. 3a, denoted as $C_{1}$ for centre bifurcation) a pair of 6-cycles always appear for $\sigma>1$, a saddle one and a second cycle (stable or unstable). A wide light blue region in the centre of Fig. 3a corresponds to the existence of an attracting 6-cycle. The two boundaries of this stability region (different from $\sigma=1$ ) correspond to two different kind of bifurcations. As proved in the following proposition, the upper boundary $C_{6}$ corresponds to a centre bifurcation of the attracting 6-cycle, while the lower boundary corresponds to a border collision bifurcation, also denoted by $B C B$ for short (as indicated in Fig. 3a), which means that a periodic point of the cycle collides with the boundary of definition of a region.

We prove now the following:

Proposition 3 Consider the system defined in (4) and (6). Let $(\sigma, \mu)$ belong to the stability region of the attracting 6-cycle born at the centre bifurcation of the fixed point $K^{*}$, then at $\mu=1+\frac{1}{\sigma^{2}}$ the 6-cycle undergoes a centre bifurcation.

To prove the proposition we recall that the attracting six cycles has two periodic points belonging to region $R_{1}$ and four periodic points belonging to region $R_{3}$. Its local stability is thus investigates as a fixed point of the map $\left(q^{\prime}, k^{\prime}\right)=\left(R_{3}\right)^{4}\left(R_{1}\right)^{2}(q, k)$ which is still a linear map, with a constant Jacobian matrix given by $\left[J_{3}\right]^{4}\left[J_{1}\right]^{2}$ where

$$
J_{3}=\left[\begin{array}{cc}
0 & 1 \\
-\sigma & 1
\end{array}\right], \quad J_{1}=\left[\begin{array}{cc}
1-\mu & 0 \\
-\sigma & 1
\end{array}\right]
$$

so that the explicit formulation is given by

$$
\left[J_{3}\right]^{4}\left[J_{1}\right]^{2}=\left[\begin{array}{ll}
\left(\sigma^{2}-\sigma\right)(1-\mu)^{2}+(2 \sigma-1)(\sigma(1-\mu)+\sigma) & -(2 \sigma-1) \\
\left(2 \sigma^{2}-\sigma\right)(1-\mu)^{2}+\left(\sigma^{2}-3 \sigma+1\right)(-\sigma(1-\mu)-\sigma) & \left(\sigma^{2}-3 \sigma+1\right)
\end{array}\right]
$$

and via algebraic computation it is easy to see that for $\mu=1+\frac{1}{\sigma^{2}}$ the two eigenvalues of this matrix are complex conjugated and equal to +1 in modulus. Thus the 6 -cycles undergoes a centre bifurcation, whose rotation number now depends on the values of the parameters $\sigma$ and $\mu$ of the system.

Figure $3 \mathrm{~b}$ shows a one-dimensional bifurcation diagram for $\sigma=1.2$ fixed and for varying $\mu$ along the vertical line shown in Fig. 3a; the wide interval of $\sigma$ values in which an attracting 6-cycle exists corresponds to the crossing of the region having triangular shape in Fig. 3a and the occurrence of a centre bifurcation (in the upper boundary of the stability region, at $\mu=1+\frac{1}{1.2^{2}}=1.69 \overline{4}$ ) and a border collision bifurcation (in the lower boundary of the stability region) is indicated.

Figure $3 \mathrm{c}$ shows a different one-dimensional bifurcation diagram for $\mu=1.5$ fixed and for varying $\sigma$ along the horizontal line shown in Fig. 3a. Again, we indicate the occurrence of the centre bifurcation of the 6 -cycle (at $\left.\sigma=\sqrt{\frac{1}{\mu-1}}=\sqrt{2}=1.4142\right)$. In addition, we note the occurrence of a (degenerate) flip bifurcation. In the following, we 
(a)

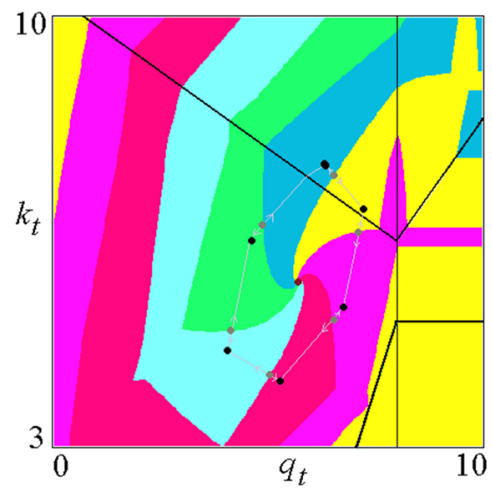

(b)

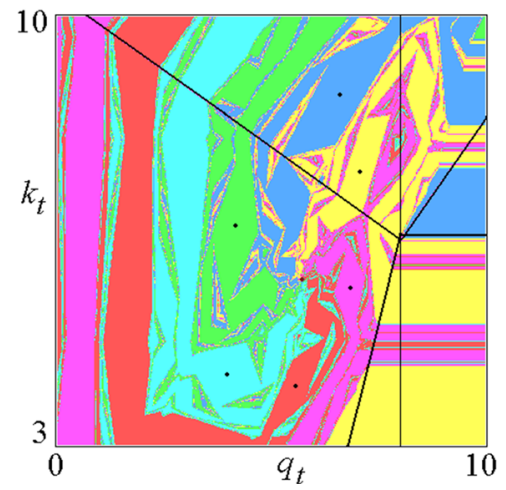

Fig. 4 Change in the basin of attractions of the six fixed points of the map $T^{6}$ at $\mu=1.5$ and $\delta=0.5$. In a $\sigma=1.1 ;$ In $\mathbf{b} \sigma=1.4$

are going to discuss these dynamic phenomena (shown in the enlargement of Fig. 3c) that are intimately related to the existence of borders in more detail.

Let us first analyze in more detail the example marked in Fig. 3c in which the attracting six cycles lose stability via the centre bifurcation commented above. Before $\sigma$ reaches the bifurcation point, the six cycles as attracting node (real eigenvalues below 1 in absolute value) and the related basins for the map $T^{6}$ have a quite simple structure (see Fig. 4a). As the parameter $\sigma$ is increased the attracting node becomes an attracting focus (complex eigenvalues below 1 in modulus), and we have a more complex structure of the basins for the map $T^{6}$ (see Fig. 4b). This is also due to the change in the partition of the phase space $\left(q_{t}, k_{t}\right)$ from 5 to 7 regions. Nevertheless, the existing attractor still is a 6-cycle; so apparently nothing has changed on the attracting set of the phase plane. However, the change of the borders of the map may cause other border collision bifurcations, which may also lead to the appearance of pairs of unstable cycles.

After the centre bifurcation of the 6-cycle, the 6-cycle continues to exist as a repelling focus, and a different attractor appears. This centre bifurcation for the fixed points of the map $T^{6}$ follows the properties recalled above (and described in Sushko and Gardini 2008), and soon after we numerically see an attracting set made up of six chaotic pieces in annular shape (see Fig. 5a). Increasing $\sigma$ the chaotic set becomes wider and the six chaotic pieces become filled (see Fig. 5b). This transition corresponds to the first homoclinic bifurcation of the 6-cycle repelling focus existing inside the annular regions (as described in Mira et al. 1996).

Increasing $\sigma$ further, a contact bifurcation between the 6 chaotic pieces and the stable set of the 6-cycle saddle (the one which was born in pair with the attracting six cycles, and which belongs to the boundaries of the six basins of the map $T^{6}$ ), occurs, a so-called contact bifurcation (Mira et al. 1996; Fournier-Prunaret et al. 1997) also known as crises (following Grebogi et al. 1983), leading to a wider chaotic area of annular shape, around the unstable fixed point (Fig. 5c). A similar transition also 
(a)

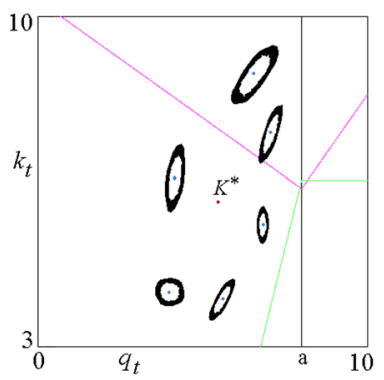

(b)

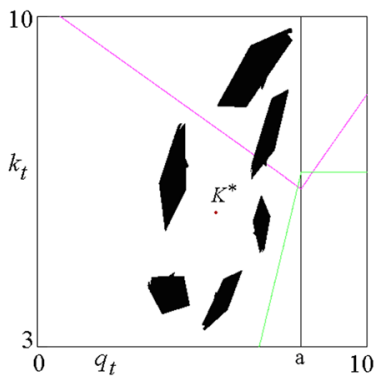

(c)

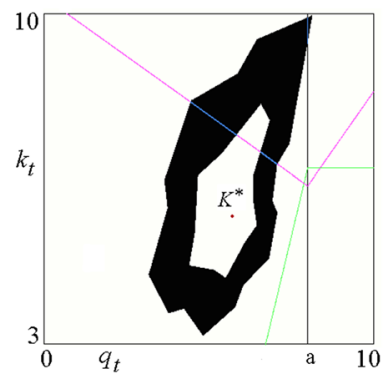

Fig. 5 Attractors of the map at $\mu=1.5$ and $\delta=0.5$. In $\mathbf{a} \sigma=1.42$ chaotic attractor in six annular regions; in $\mathbf{b} \sigma=1.454$ six chaotic pieces; in $\mathbf{c} \sigma=1.465$ one annular region

occurs along the other path shown in Fig. 3b, at fixed $\sigma=1.2$ and increasing $\mu$ after the centre bifurcation of the 6-cycle, an example is shown in Fig. 6a at $\mu=2$.

It is interesting to note that in the piecewise linear case, the boundaries of the chaotic areas are perfectly known. In fact, a chaotic area can exist only if a border is crossed by some pieces of a chaotic attracting set, and the intersection of the invariant chaotic set with the borders of definition of the map gives a so-called generating arc (see Gardini 1994; Mira et al. 1996), whose images under $T$ give the boundaries of the chaotic sets. In the example shown in Fig. $5 \mathrm{c}$ the generating arc consists of two segments belonging to the boundary between the regions $R_{3}$ and $R_{1}$, while in the example shown in Fig. 6 a (where the annular area is larger) the generating arc $(g)$ consists in one single segment, resulting from the intersection of the invariant area with the border between the regions $R_{3}$ and $R_{1}$. With 6 iterates of the segment $(g)$ the external boundary of the annular area is obtained, and a small piece of the internal boundary also (see the black segments in Fig. 6b), while with 6 more images also the internal boundary is completely obtained (see the red segments in Fig. 6b). In addition, as the parameter $\mu$ is further increased, the unstable fixed point $K^{*}$ may also undergo its first homoclinic bifurcation leading to a chaotic area in one piece including the unstable fixed point. As is well known, this occurs when all these images of the "generating arcs" cross the fixed point, leading to a snap-back repeller (so-called after Marotto 1978, 2005 see also Gardini 1994; Gardini et al. 2011). This bifurcation is shown in Fig. 6d. The first six iterates of the generating arc $(g)$ gives the external boundary of the one-piece chaotic area, and the seventh iterate (shown in blue) cross through the fixed point $K^{*}$ (and thus all the further iterates also), thus leading to a closed chaotic area, as shown in Fig. 6c, no longer annular, as it includes the fixed point $K^{*}$ now also homoclinic.

Returning to Fig. 5, we can interpret the homoclinic bifurcation of the 6-cycle shown in Fig. 5b in a similar way: The images of the generating $\operatorname{arc}\left(\right.$ for the map $T^{6}$ ) give the boundaries of the six chaotic pieces, and when these images cross through the period- 6 repelling focus, the six areas are closed and the period- 6 repelling focus becomes a snap-back repeller (i.e. with homoclinic orbits). After that, as commented above, the reunion of the six chaotic pieces into one occurs due to an homoclinic bifurcation of the saddle 6-cycle. 
(a)

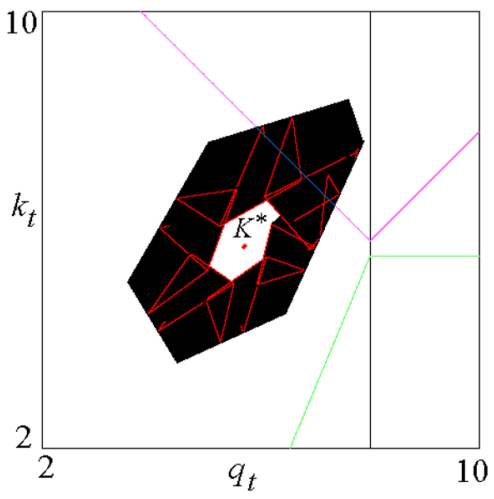

(c)

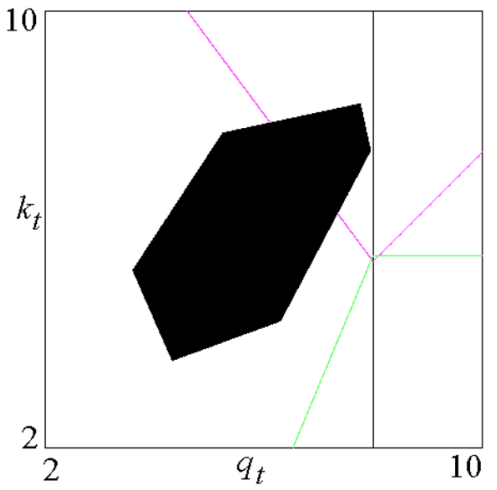

(b)

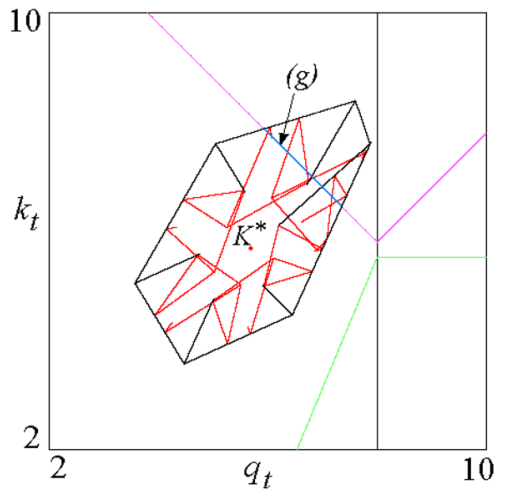

(d)

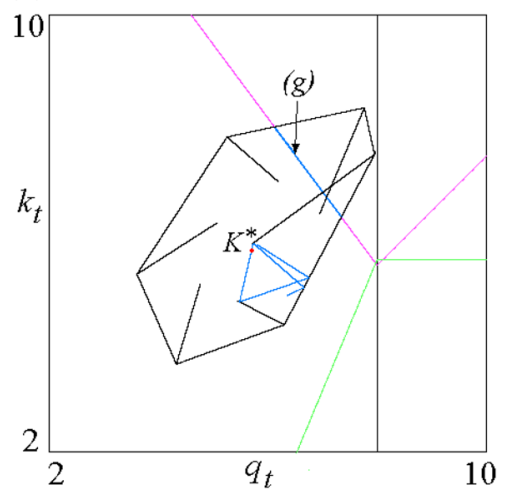

Fig. 6 Chaotic areas of the map at $\delta=0.5$ and $\sigma=1.2$. In $\mathbf{a}$ at $\mu=2$ chaotic annual area; in $\mathbf{b}$ the generating segment $(g)$ and its iterates giving the boundary of the area in (a). In $\mathbf{c}$ at $\mu=2.348$ chaotic area; in $\mathbf{d}$ the generating segment $(g)$ and its iterates giving the external boundary of the one-piece area in (d) (color figure online)

As a last point, we note that the existence of such homoclinic cycles leads to more complicated dynamics. Figure 7a shows a trajectory of $q_{t}$ when the attractor is the annular chaotic area of Figs. 6a, 7b shows a trajectory $q_{t}$ after the homoclinic bifurcation of the fixed point $K^{*}$ when there is a one-piece chaotic area without holes. An increase in unpredictability can be observed. In fact, in Fig. 7a, the trajectory is confined to the annular region and the quantity capacity points travel around the (unstable) fixed point without approaching it, while in Fig. $7 \mathrm{~b}$ the trajectory approaches the (unstable) equilibrium from time to time, in an unpredictable way.

\subsection{Border collision bifurcations and degenerate flip bifurcation}

In this section we first analyze the effects of a border collision bifurcation, i.e. bifurcation that occurs if a point of the attractor collides with one of the borders. This type 
(a)

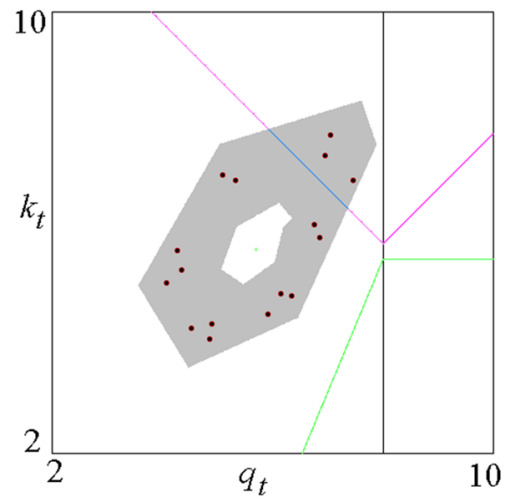

(b)

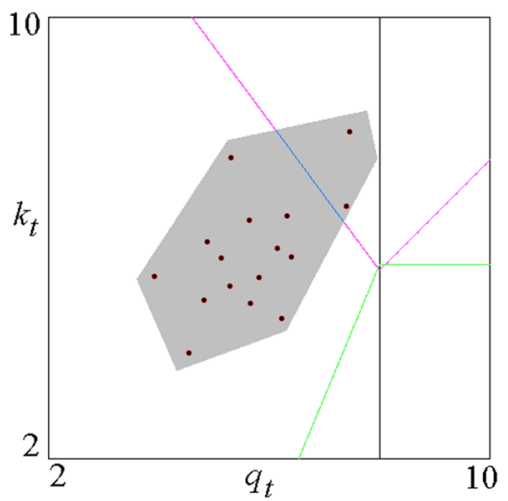

Fig. 7 A few iterations of a trajectory of the map at $\delta=0.5$ and $\sigma=1.2$. In a at $\mu=2$ as in Fig. 6a; in $\mathbf{b}$ at $\mu=2.348$ as in Fig. $6 \mathrm{c}$

(a)

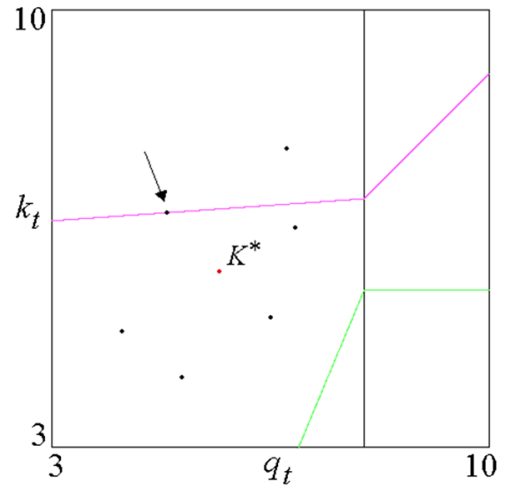

(b)

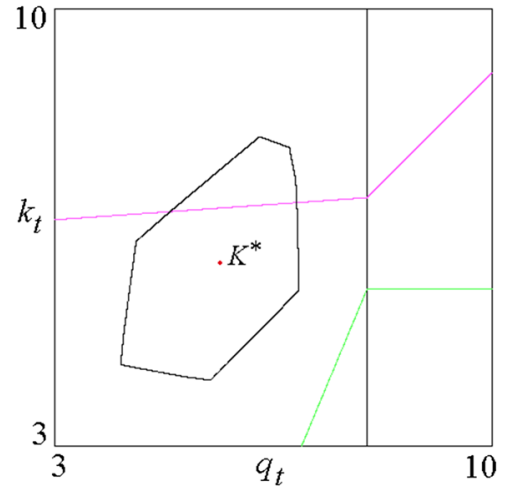

Fig. 8 In a border collision bifurcation of the 6-cycle at $\delta=0.5, \sigma=1.2$ and $\mu=0.9292$. In $\mathbf{b}$ closed invariant curve existing as attracting set at $\mu=0.9291$

of bifurcation is quite common in piecewise smooth systems, and we shall see that different dynamic behaviours may be associated with a border collision of some cycle.

We start with the example shown in the bifurcation diagram for $\sigma=1.2$ fixed and varying $\mu$ along the vertical line shown in Fig. 3a. Starting from a $\mu$ value inside the range in which the 6-cycle is stable, decreasing $\mu$ a lower bifurcation value is met, at which a border collision bifurcation occurs leading to the disappearance of the 6-cycle (in contrast to the centre bifurcation discussed above at which the 6-cycle continues to exist but becomes a repelling focus). In Fig. 8a we illustrate the 6-cycle at the border collision. This type of bifurcation occurs if one of the periodic points of the 6-cycle belongs to the border separating the regions $R_{3}$ and $R_{1}$, i.e. the border associated with the capacity constraint. In this example, the bifurcation leads to the disappearance of the 6-cycle, and the attracting set existing after the bifurcation (detected numerically) 
involves quasiperiodic trajectories belonging to a closed attracting curve, as shown in Fig. 8b.

In general it is difficult to predict what happens after a border collision bifurcation. The existence of a cycle is strictly related with the sequence of maps which are applied in order to get the cycle. Thus whenever a periodic point merges with a border, and a border collision occurs, we may have some change in the attracting set. As we have just seen, this change may lead to the disappearance of one attractor and to the birth of a different one. However, it may also be the case that the cycle continues to exist with the same periodicity, and that only the definitions of the involved maps have changed.

Also in the other bifurcation diagram shown in Fig. 3c, for $\mu=1.5$ fixed and varying $\sigma$ along the horizontal path, we can see two more examples of border collision bifurcation there indicated with the points $B_{1}$ and $B_{2}$. At $\sigma=3$ an attracting 7-cycle exists, and decreasing $\sigma$ we can see that the position of the points of the attracting 7 -cycle changes a little. In Fig. 9 a we represent the attractor at the point $B_{2}$; the 7-cycle has one of the periodic points on the border between the regions $R_{3}$ and $R_{5}$, and we can also notice that one point of the cycle is in region $R_{2}$. The effect of this border collision bifurcation is a kind of persistence. That is, also after the bifurcation the attractor is a cycle of the same period, but the periodic point which crosses from region $R_{5}$ to region $R_{3}$ causes a marked change also to the position of the other periodic points, as the sequence of functions which are now applied to the points is now different. In Fig. 9b, we can see, as $\sigma$ is slightly decreased, how the periodic points of the 7-cycle have changed their position in the different regions, in particular, now no point of the cycle belongs to region $R_{2}$.

Less visible is the effect of the border collision bifurcation that occurs as $\sigma$ is further decreased, at the point $B_{1}$. The same periodic point which crossed the boundary approaches the other boundary between the regions $R_{3}$ and $R_{1}$, as shown in Fig. 9c, and then it crosses the border. However, the effect now is smoother, since the attractor persist as a 7-cycle with the transition of one periodic point from region $R_{3}$ to region $R_{1}$.

We remark that at the point $P$ in Fig. 3c no bifurcation occurs. This peculiar point in the bifurcation diagram is due to the particular position of the 7 periodic points in the phase space, having 5 points on the same vertical line, as shown in Fig. 9d.

Our discussion thus shows that border collision bifurcations may be "dangerous" in the sense that it is difficult to predict what happens after the bifurcation (it is difficult to predict whether the bifurcation involves the appearance of a new attractor or whether it only modifies the shape of the attractor).

Finally we show that due to the existence of borders not only new types of bifurcation can occur such as the discussed centre bifurcations and border collision bifurcations, but that also a well known bifurcation type such as the flip bifurcation is modified to a so-called "degenerate flip bifurcation" (see Sushko and Gardini 2010). In fact, in piecewise linear maps at the flip bifurcation value there exist segments filled with cycles of doubled period, and the length of the segments, along the invariant eigenvector of the cycle undergoing the flip bifurcation, depends on the distance of the periodic points from the borders of definition for the map.

An example is shown in the one-dimensional bifurcation diagram in Fig. 3c at $\sigma \approx 2.17$ indicated as "Flip" and the related enlargement. Decreasing $\sigma$, the 7 -cycle 
(a)

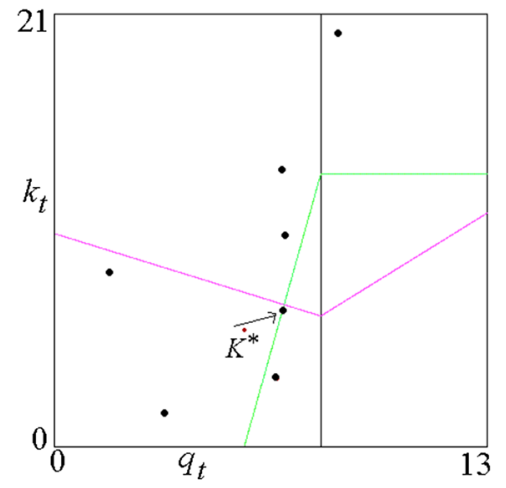

(c)

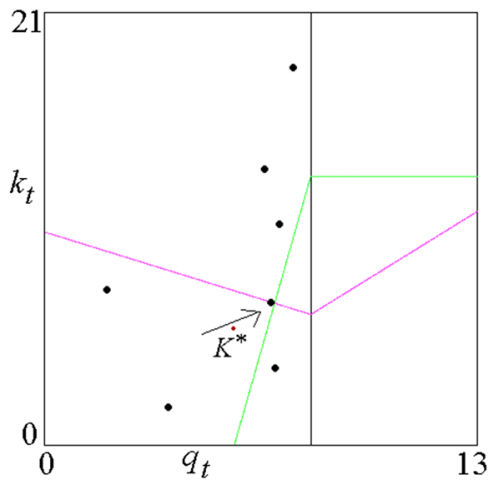

(b)

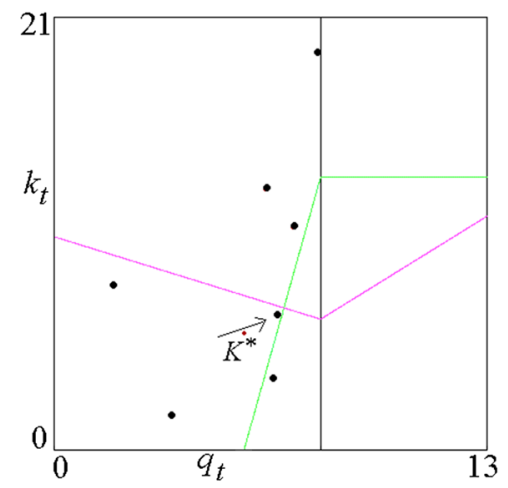

(d)

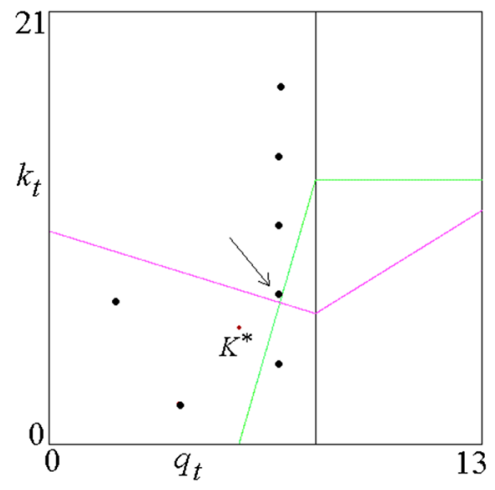

Fig. 9 Period-7 attactors at $\delta=0.5$ and $\mu=1.5$. In $\mathbf{a} \sigma=2.8758$ at the bifurcation B1. In $\mathbf{b} \sigma=2.8757$. In $\mathbf{c} \sigma=2.84$ at the bifurcation B2. In $\mathbf{d} \sigma=2.79$

(as shown in Fig. 10) loses stability via an eigenvalue that crosses the value -1 giving rise to a cycle of double period (i.e. of period 14 in our case). However, at the bifurcation value there exist a segment filled with cycles of period 14, and the length of the segment depends on the distance of the periodic points from the borders. In our example one periodic point is very close to the vertical boundary $(q=a)$, as shown in Fig. 10a. Thus the segments are very short, and after the bifurcation the two points associated with that periodic point are on opposite side with respect to the boundary $q=a$, as shown in Fig. 10b.

\section{Conclusions}

Models with constraints and boundaries have become increasingly popular in the aftermath of the financial crisis. The intention of this paper was to show the impact that these constraints may impinge upon the dynamic out-of-equilibrium processes. In order to avoid any ambiguity, we on purpose specified all functional relationships as 
(a)

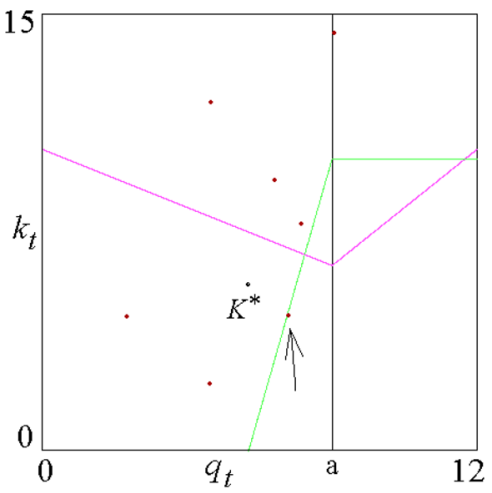

(b)

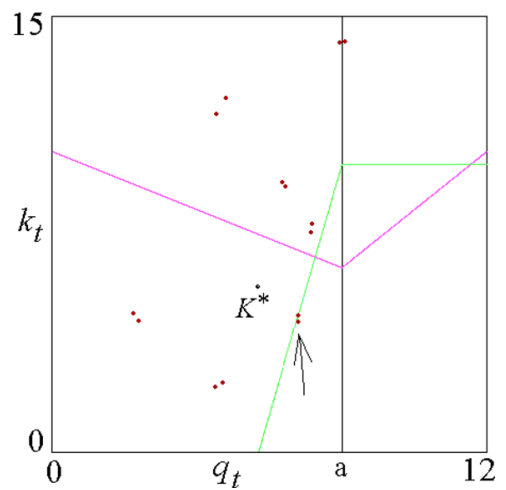

Fig. 10 Degenerate flip bifurcation of the map at $\delta=0.5$ and $\mu=1.5$. In $\mathbf{a} \sigma=2.18$ close to the bifurcation, 7-cycle. In $\mathbf{b} \sigma=2.15$ after the bifurcation, 14-cycle

linear, leaving the constraints as the only source for complex dynamics. We consider this specification as being of particular importance, since economists appear to agree more easily on the nature and specification of borders (some of them are actually intrinsic to the definition of the variables themselves) than on specification of a nonlinear out-of-equilibrium dynamics. As example, we constructed a very simply model of quantity and capactity dynamics in a Marshallian tradition, in which we account explicitly for intrinsic boundaries: a non-negativity constraint on price, quantity and capacity; a capacity constraint on output and a downward rigidity (depreciation) on capacity. We showed that these model elements are sufficient to produce cyclical phenomena as well as more complex dynamic behaviours.

The resulting piecewise linear model has a unique fixed point which loses stability via a centre bifurcation, giving rise to a (stable) 6-cycle, which in turn loses stability via another (secondary) centre bifurcation, resulting in a chaotic cycle consisting of six annual pieces, that eventually merge to one annular attractor and finally to a one piece chaotic attractor. Due to the sensitivity on initial conditions the dynamic path on a chaotic attractor is unpredictable. However, the boundaries and constraints not only give rise to these complex dynamic patterns, they also shape the attractors. The intersection of the attractor with the borders leads to generating arcs whose images form the outer boundary of the attractor and —in the case of annual attractor-also the inner boundary.

In addition, we showed border collision bifurcations, which occur if a point of the time path merges with a boundary, and we stressed the "dangerous" nature of such border collision bifurcations (in the sense that it is not easy to predict whether a qualitative change in the attractor happens or not). Finally, we show the degenerate nature of the occurring flip bifurcations.

Given the linear specification of our model, borders and constraints alone are responsible for the complex dynamics. Note that specifying nonlinear functional forms would introduce another source for such a behaviour. Thus, cyclical or complex dynamics should not be considered as an exceptional case. Instead, it seems to be intrinsic in 
economic models, a fact that deserves more attention after the financial crisis has highlighted the inherent instability of economic processes.

Acknowledgements The authors would like to thank two anonymous referees for valuable suggestions and for the extremely careful reading. Ingrid Kubin would like to thank WU Vienna University of Economics and Business and the Austrian National Bank for financial support; and the University of Urbino (It) for the kind hospitality and fruitful discussions during her research visiting period.

Funding Open access funding provided by Vienna University of Economics and Business (WU).

Open Access This article is licensed under a Creative Commons Attribution 4.0 International License, which permits use, sharing, adaptation, distribution and reproduction in any medium or format, as long as you give appropriate credit to the original author(s) and the source, provide a link to the Creative Commons licence, and indicate if changes were made. The images or other third party material in this article are included in the article's Creative Commons licence, unless indicated otherwise in a credit line to the material. If material is not included in the article's Creative Commons licence and your intended use is not permitted by statutory regulation or exceeds the permitted use, you will need to obtain permission directly from the copyright holder. To view a copy of this licence, visit http://creativecommons.org/licenses/by/4.0/.

\section{References}

Agliari A, Commendatore P, Foroni I, Kubin I (2011) Border collision bifurcations in a footloose capital model with first nature firms. Comput Econ 38:349-366

Aramburo SA, Castaneda Acevedo JA, Moralesa YA (2012) Laboratory experiments in the system dynamics field. Syst Dyn Rev 28:94-106

Barnett WA, Serletis A, Serletis D (2015) Nonlinear and complex dynamics in economics. Macroecon Dyn 19:1749-1779

Bischi G, Lamantia F (2012) Routes to complexity induced by constraints in Cournot oligopoly games with linear reaction functions. Stud Nonlinear Dyn Econom 16:1-30

Bischi G, Lamantia F, Sushko I (2012) Border Collisopn Bifurcation in a simple oligopoly model with constraints. Int J Appl Math Stat 26:121-135

Boehm V, Kaas L (2000) Differential savings, factor shares and endogenous growth cycles. J Econ Dyn Control 24:965-980

Caballé J, Jarqueb X, Michetti E (2006) Chaotic dynamics in credit constrained emerging economies. J Econ Dyn Control 30:1261-1275

Commendatore P, Currie M, Kubin I (2008) Footloose entrepreneurs, taxes and subsidies. Spat Econ Anal 3:115-141

Commendatore P, Kubin I, Petraglia C (2008) Productive public expenditure in a new economic geography model. Écon Int 114:133-160

Commendatore P, Kubin I, Sushko I (2020) A propos brexit: on the breaking up of integration areas-an NEG analysis. Spat Econ Anal (forthcoming). https://doi.org/10.1080/17421772.2019.1701702

Currie M, Kubin I (1997) Investment in fixed capital and competitive industry dynamics. Oxf Econ Pap 49:521-542

Currie M, Kubin I (2006) Chaos in the core periphery model. J Econ Behav Organ 60:252-275

Dosi G, Gary MS, Lovallo D (2008) Boom and bust behavior: on the persistence of strategic decision Biases. In: Hodgkinson GP, Starbuck WH (eds) The Oxford handbook of organizational decision M. Oxford University Press, Oxford

Fournier-Prunaret D, Mira C, Gardini L (1997) Some contact bifurcations in two-dimensional examples. Grazer Math Ber 334:77-96

Gardini L (1992) Some global bifurcations of two-dimensional endomorphisms by use of critical lines. Nonlinear Anal, Theory, Methods Appl 18:361-399

Gardini L (1994) Homoclinic bifurcations in $n$-dimensional endomorphisms, due to expanding periodic points. Nonlinear Anal, Theory Methods Appl 23:1039-1089

Gardini L, Puu T, Sushko I (2006) The Hicksian model with investment floor and income ceiling. In: Puu T, Sushko I (eds) Business cycle dynamics_-models and tools. Springer, Berlin, pp 179-192 
Gardini L, Sushko I, Avrutin V, Schanz M (2011) Critical homoclinic orbits lead to snap-back repellers. Chaos Solitons Fractals 44:433-449

Gardini L, Sushko I, Matsuyama K (2018) 2D discontinuous piecewise linear map: emergence of fashion cycles fashion model. Chaos: Interdiscip J Nonlinear Sci 28(5):055917. https://doi.org/10.1063/1. 5018588

Gomes O (2006) Routes to chaos in macroeconomic theory. J Econ Stud 33:437-468

Grandmont JM (2008) Nonlinear difference equations, bifurcations and chaos: an introduction. Res Econ 62:122-177

Grebogi C, Ott E, Yorke JA (1983) Crisis: sudden changes in chaotic attractors and transient chaos. Phys D 7:181

Gumowski I, Mira C (1980) Dynamique chaotique. Cepadues Editions, Toulouse

Hommes C (1995) A reconsideration of Hicks non-linear trade cycle model. Struct Change Econ Dyn $6: 435-459$

Kaas L, Zink S (2007) Human capital and growth cycles. Econ Theory 31:19-33

Kopel M (1996) Periodic and chaotic behaviour of a simple R\&D model. Ric Econ 50:235-265

Kubin I, Gardini L (2013) Border collision bifurcations in boom and bust cycles. J Evol Econ 23:811-829

Kubin I, Zörner T (2019) Credit cycles, human capital and the distribution of income. JEBO (in press). https://doi.org/10.1016/j.jebo.2019.02.022

Laugesen J, Mosekilde E (2006) Border-collision bifurcations in a dynamic management game. Comput Oper Res 22:464-478

Maistrenko YL, Sushko IM, Gardini L (1998) About two mechanisms of reunion of chaotic attractors. Chaos, Solitons Fractals 9:1373-1390

Marotto FR (1978) Snap-back repellers imply chaos in Rn. J Math Anal Appl 63:199-223

Marotto FR (2005) On redefining a snap-back repeller. Chaos, Solitons Fractals 25:25-28

Matsuyama K (1999) Growing through cycles. Econometrica 67:335-347

Matsuyama K (2007) Credit traps and credit cycles. AER 97:503-516

Matsuyama K (2013) The good, the bad, and the ugly: an inquiry into the causes and nature of credit cycles. Theor Econ 8:623-51

Mira C, Gardini L, Barugola A, Cathala JC (1996) Chaotic dynamics in two-dimensional nonivertible maps. World Scientific, Singapore

Nusse HE, Yorke JA (1992) Border-collision bifurcations including period two to period three for piecewise smooth systems. Phys. D 57:39-57

Rendahl P (2017) Inequality constraints and Euler equation based solution methods. Econ J 125:1110-1135

Schmitt N, Tuinstra J, Westerhoff F (2017) Side effects of nonlinear profit taxes in an evolutionary market entry model: abrupt changes, coexisting attractors and hysteresis problems. J Econ Behav Organ 135:15-38

Schmitt N, Tuinstra J, Westerhoff F (2018) Stability and welfare effects of profit taxes within an evolutionary market interaction model. Rev Int Econ 26:691-708

Sterman JD (1989) Misperceptions of feedback in dynamic decision making. Org Behav Human Decis Process 43:301-335

Sterman JD, Henderson R, Beinhocker ED, Newman LI (2007) Getting big too fast: strategic dynamics with increasing returns and bounded rationality. Manag Sci 53:683-696

Sushko I, Avrutin V, Gardini L (2015) Bifurcation structure in the skew tent map and its application as a border collision normal form. J Differ Equ Appl. https://doi.org/10.1080/10236198.2015.1113273

Sushko I, Gardini L (2008) Center bifurcation for a two-dimensional border-collision normal Form. Int J Bifurc Chaos 18:1029-1050

Sushko I, Gardini L (2010) Degenerate bifurcations and border collisions in piecewise smooth 1D and 2D maps. Int J Bifurc Chaos 20:2045-2070

Sushko IM, Maistrenko YL, Gardini L (1999) On chaotic attractors at the transition from homeomorphism to endomorphism in a family of two-dimensional piecewise linear maps. Grazer Math Ber 339:335-346

Sushko I, Gardini L, Avrutin V (2016) Nonsmooth one-dimensional maps: some basic concepts and definitions. J Differ Equ Appl 1-55. https://doi.org/10.1080/10236198.2016.1248426

Sushko I, Gardini L, Matsuyama K (2014) Superstable credit cycles and U-sequence. Chaos Solitons Fractals 59:13-27

Sushko I, Gardini L, Matsuyama K (2016) Robust chaos in a credit cycle model defined by a one-dimensional piecewise smooth map. Chaos Solitons Fractals 91:299-309 
Sushko I, Gardini L, Matsuyama K (2017) Coupled chaotic fluctuations in a model of international trade and innovation: some preliminary results. Commun Nonlinear Sci Numer Simul 58:287-302

Sushko I, Gardini L, Puu T (2010) Regular and chaotic growth in a Hicksian floor/ceiling model. J Econ Behav Organ 75:77-94

Sushko I, Puu T, Gardini L (2003) The Hicksian floor-roof model for two regions linked by interregional trade. Chaos, Solitons Fractals 18:593-612

Tramontana F, Gardini L, Agliari A (2011a) Endogenous cycles in discontinuous growth models. Math Comput Simul 81:1625-1639

Tramontana F, Gardini L, Ferri P (2010) The dynamics of the NAIRU model with two switching regimes. J Econ Dyn Control 34:681-695

Tramontana F, Westerhoff F, Gardini L (2011b) Heterogeneous speculators and asset price dynamics: further results from a one-dimensional discontinuous piecewise-linear model. Comput Econ 38:329-347

Tversky A, Kahneman D (1974) Judgment under uncertainty: heuristics and biases. Sci, New Ser 185:11241131

Publisher's Note Springer Nature remains neutral with regard to jurisdictional claims in published maps and institutional affiliations. 